\title{
SPECTRAL SYMMETRY OF THE DIRAC OPERATOR IN THE PRESENCE OF A GROUP ACTION
}

\author{
H. D. FEGAN AND B. STEER
}

\begin{abstract}
Let $G$ be a compact Lie group of rank two or greater which acts on a spin manifold $M$ of dimension $4 k+3$ through isometries with finite isotropy subgroups at each point. Define the Dirac operator, $P$, on $M$ relative to the split connection. Then we show that $P$ has spectral $G$-symmetry. This is first established in a number of special cases which are both of interest in their own right and necessary to establish the more general case. Finally we consider changing the connection and show that for the Levi-Civita connection the equivariant eta function evaluated at zero is constant on $G$.
\end{abstract}

\section{INTRODUCTION}

For a selfadjoint elliptic operator $D$ on a compact Riemannian manifold $M$ the eta function $\eta(s)$ is defined as

$$
\eta(s)=\sum_{\lambda \in \sigma^{\prime}(D)} \operatorname{sign} \lambda|\lambda|^{-s}, \quad \operatorname{re}(s)>\operatorname{dim} M,
$$

where $\sigma(D)$ is the spectrum of $D$ and $\sigma^{\prime}(D)=\sigma D-\{0\}$. When a compact group $G$ acts, and $D$ is equivariant, then each eigenspace $V_{\lambda}(\lambda \neq 0)$ is a finite-dimensional $G$-space, and one sets

$$
\eta(g, s)=\sum_{\lambda \in \sigma^{\prime}(D)} \operatorname{tr}\left(g \mid V_{\lambda}\right) \operatorname{sign} \lambda|\lambda|^{-s} .
$$

This function is defined and discussed in the series of papers [2] where it is shown that if $D$ is an operator like the Dirac operator, then $\eta(g, s)$ has a meromorphic extension to the whole of $\mathbf{C}$; one, moreover, which is holomorphic at 0 . Now, we suppress $g$ from the notation and think of $\eta(0) \in R(G) \otimes_{\mathbf{Z}} \mathbf{R}$, the character ring of $G$ with coefficients in $\mathbf{R}$. (For operators like the Dirac operator, the value at 0 of the eta function is real.)

On an odd-dimensional spin manifold, the value $\eta(0) \bmod \mathbf{Z}$ for the Dirac operator is an analogue of the $\hat{A}$ genus for even-dimensional spin manifolds. It vanishes for manifolds of dimension $4 k+1$ for (trivial) algebraic reasons, but in dimensions $4 k+3$ it is a rather delicate invariant. One cannot really expect that it should vanish if there is an $S^{1}$ action in the way that the $\hat{A}$ genus does [2]. (Indeed for quotients of $P S L_{2}(\mathbf{R})$ we know that it does not [10] and that $\eta(0)$ as a rational character is not constant.) One can vary the Dirac operator,

Received by the editors November 10, 1989 and, in revised form, October 23, 1990.

1980 Mathematics Subject Classification (1985 Revision). Primary 58G25. 
not just by altering the metric, but by changing the connection through metric preserving ones, as for example in [11]. When there is an action of a Lie group $G$ of rank $\geq 2$ we are able to prove a number of vanishing theorems. The main result is the following.

Theorem 1.1. If $G$, a compact Lie group, acts on the spin manifold $M^{4 k+3}$ through isometries, and with finite isotropy subgroups at every point, then for a certain connection, the split connection, the spectrum of the Dirac operator $P$ is $G$-symmetric if rank $G \geq 2$.

This main result is proved by establishing a number of special cases. When taken together these are equivalent to Theorem 1.1.

The first of these special cases is when $M$ is the quotient of $G$ by a discrete subgroup $\Gamma: M=\Gamma \backslash G$. While this case is eventually subsumed by another special case, it is both necessary in order to prove this other case and also of interest in its own right.

Theorem 1.2. If $G$ is a compact Lie group of rank $\geq 2$ and $\Gamma$ is any discrete subgroup then $\eta_{\Gamma \backslash G}(s)=0$.

Here we have stated the result using the eta function, $\eta_{\Gamma \backslash G}$, of the Dirac operator $P$ on $\Gamma \backslash G$ which is defined in equation (1.1). This result is proved in $\S 2$ where it appears as Theorem 2.6.

It is a surprising fact while Theorem 1.1 holds when $G$ is an abelian group or a nonabelian group, neither of these cases can be deduced from the other and a different proof is used for each case. The reason why the case of a nonabelian group $G$ does not establish that for its maximal torus $T$ is that a connection which is split for $G$ may not be split for $T$. For example, Theorem 1.2 holds using the Levi-Civita connection on $G$ and the Levi-Civita connection is decidedly not split for $T$. The essential difference between the nonabelian and abelian cases is that, with respect to the left invariant trivialization, the Dirac operator has a nonzero constant term in the nonabelian case but not in the abelian one.

The abelian case is studied in $\S 4$ and the nonabelian case in $\S 5$. In both cases, the statement of the result is the same. The space of spinors is decomposed into finite dimensional invariant subspaces $S_{\theta, \lambda}$. These are invariant under both the group action and the Dirac operator $P$. The result is that $P$ restricted to act on $S_{\theta, \lambda}$ has spectral symmetry.

Theorem 1.3. The operator $P$ restricted to act on $S_{\theta, \lambda}$ has two eigenvalues $\mu$ and $-\mu$ and these have the same multiplicity.

This result is proved as Theorem 4.5 in the abelian case and Theorem 5.10 in the nonabelian case. A precise description of $S_{\theta, \lambda}$ is somewhat involved and is left until later in the paper.

The final section, $\S 6$, is concerned with changing the connection. If $\widetilde{\nabla}$ is a connection which preserves the metric, $\widetilde{P}$ is the corresponding Dirac operator and $\tilde{\eta}$ the corresponding eta function, then we can compare these to the split ones. As noted earlier, the equivariant eta function of equation (1.2) defines an eta invariant in the ring $R(G) \otimes_{\mathbf{Z}} \mathbf{R}$. Our result is that modulo representations this is independent of the choice of connection. 
Theorem 1.4. Reduced mod $R(G)$, that is an element of $R(G) \otimes \mathbf{R} / R(G)$, $\tilde{\eta}_{G}(o, g)$ is constant.

This is proved as Corollary 6.5 .

To complete the paper is an appendix on the rank one case. Many of the details of the rank one case have been studied earlier, see [5] for the compact case and [10] for the noncompact case. However, this case is interesting, especially to physicists, in its own right and shows the necessity of the restriction rank $G \geq 2$ for our results. We show by an explicit calculation that Theorem 1.2 holds for $G=S U(2)$ and $\Gamma=\{I\}$ but does not hold for $G=S U(2)$ and $\Gamma=\{I,-I\}$ when $\Gamma \backslash G=S O(3)$.

The first author was supported by an Efroymson Memorial Lectureship.

\section{SPECTRAL SYMMETRY ON A COMPACT GROUP}

Let $G$ be a compact nonabelian Lie group with Lie algebra $\mathscr{G}$ and let $\rho$ be a bi-invariant metric. Let $\nabla$ be the corresponding Levi-Civita connection on $G$. Set $\chi=\Delta \circ \widetilde{a d}$ where $\Delta: \operatorname{spin}(\mathscr{G}) \rightarrow$ End $S$ is the spin representation on Lie algebras and $\widetilde{a d}: G \rightarrow \operatorname{spin}(\mathscr{G})$ is the lift of the adjoint representation. If $G$ is not simply connected there may be several spin structures. We use $\mathbf{S}$ to denote the trivial spin bundle over $G$, given by identifying $T(G)$ with $\mathscr{G} \times G$ by left translation. Then we may identify

$$
C^{\infty}(\mathbf{S})=C^{\infty}(G) \otimes S,
$$

and lift the connection $\nabla$ to $\mathbf{S}$ in the natural way. The Lie algebra $\mathscr{G}$ is contained in the Clifford algebra $\operatorname{Cliff}(\mathscr{G})$ (defined so $X Y+Y X=-2 \rho(X, Y)$ ) which acts on $S$ by Clifford multiplication. Pick an orthonormal basis $E_{1}, \ldots$, $E_{r}$ for $\mathscr{G}$.

Lemma 2.1. (i) $\chi(X)=-\frac{1}{4} \sum\left[X, E_{i}\right] E_{i}$,

(ii) $\nabla_{E_{j}} \psi=-\frac{1}{8} \sum\left[E_{j}, E_{i}\right] E_{i} \psi$,

where the sums are over the index $i$ which runs from 1 to $r$ and $\psi$ is a basic spinor.

Proof. For (i) we observe that since $\widetilde{a d}$ is a lifting of ad we have

$$
\chi(X) Y-Y \chi(X)=[X, Y] .
$$

A direct calculation now gives the result as in [3]. If $\omega_{i j}$ is the matrix of $\nabla$ (so that $\left.\nabla_{X} E_{i}=\sum \omega_{i j}(X) E_{j}\right)$ then $\nabla \psi=-\frac{1}{4} \sum \omega_{i j} E_{i} E_{j} \psi$, see [5]. Thus using the well-known fact that $\nabla_{X} Y=\frac{1}{2}[X, Y]$ we have (ii).

The Dirac operator is defined by

$$
P \varphi=\sum \omega E_{i} \nabla_{E_{i}} \varphi \in C^{\infty}(\mathbf{S}),
$$

where $\omega=i^{(r+1)(r+2) / 2} E_{1} \cdots E_{r}$ is the volume element and the multiplication is Clifford multiplication. Choose a spinor basis for $S$ corresponding to the basis $E_{1}, \ldots, E_{r}$ for $\mathscr{G}$. The computation of $P$ can be carried out in terms of that of $P \psi$ for a basic spinor $\psi=1 \otimes S$ since

$$
P(f \otimes s)=\sum \nu\left(E_{i}\right) f \otimes \omega E_{i} s+f P \psi .
$$


Here $\nu\left(E_{i}\right) f$ is the directional or Lie derivative of $f$ in the direction of the left invariant field given by $E_{i}$. We shall also write $L_{E_{i}} f$ for $\nu\left(E_{i}\right) f$. Now make the following definitions:

$$
\begin{aligned}
Q & =\sum \nu\left(E_{i}\right) \otimes \omega E_{i} \quad \text { and } \\
M & =\sum \omega E_{i} \chi\left(E_{i}\right)
\end{aligned}
$$

so $Q: C^{\infty}(\mathbf{S}) \rightarrow C^{\infty}(\mathbf{S})$ and $M: S \rightarrow S$.

Proposition 2.2. For a basic spinor $\psi, P \psi=\frac{1}{2}(1 \otimes M) \psi$.

Proof. From Lemma 2.1 we have

$$
\nabla_{E_{j}} \psi=\frac{1}{2} \chi\left(E_{j}\right) \psi
$$

and the result of the proposition now follows immediately.

Corollary 2.3. $P=Q+\frac{1}{2}(1 \otimes M)$.

Before proceeding we introduce the following notation: the anticommutator $A B+B A$ of two operators is denoted by $\{A, B\}$; the Casimir operator $-\sum \nu\left(E_{i}\right)^{2}$ by $\Omega$. Choose a simple system of roots for $\mathscr{G}$ and let $\rho=\frac{1}{2} \sum \alpha>0 \alpha$ be half the sum of the positive roots. Finally set

$$
R=\sum \nu\left(E_{i}\right) \otimes \chi\left(E_{i}\right)
$$

Lemma 2.4. (i) $Q^{2}=\nu(\Omega) \otimes 1+2 R$,

(ii) $M^{2}=9\|\rho\|^{2}$,

(iii) $\{Q, 1 \otimes M\}=-6 R$,

(iv) $(1 \otimes M) R=R(1 \otimes M)$,

(v) $Q R=R Q$,

(vi) $\chi(X) M=M_{\chi}(X)$ for all $X \in \mathscr{G}$.

Proof. Each part is proved by a simple direct calculation. Note that the power $(r+1)(r+2) / 2$ of $i$ in $\omega$ results in $\omega E \omega=E$ for any basis vector $E$, which in turn implies $\omega^{2}=(-1)^{r-1}$. We verify part (ii) as an illustration.

$$
\begin{aligned}
M^{2} & =\sum \omega E_{i} \chi\left(E_{i}\right) \omega E_{j} \chi\left(E_{j}\right)=\sum E_{i} \chi\left(E_{i}\right) E_{j} \chi\left(E_{j}\right) \\
& =\frac{1}{2} \sum\left(E_{i} E_{j} \chi\left(E_{i}\right) \chi\left(E_{j}\right)+E_{j} E_{i} \chi\left(E_{j}\right) \chi\left(E_{i}\right)+\sum E_{i}\left[E_{i}, E_{j}\right] \chi\left(E_{j}\right)\right) \\
& =\frac{1}{2}\left\{\sum E_{i} E_{j}\left(\chi\left(E_{i}\right) \chi\left(E_{j}\right)-\chi\left(E_{j}\right) \chi\left(E_{i}\right)\right)-2 \sum \chi\left(E_{i}\right)^{2}\right\}-4 \sum \chi\left(E_{j}\right)^{2} \\
& =\frac{1}{2} \sum E_{i} E_{j} \chi\left(\left[E_{i}, E_{j}\right]\right)-5 \sum \chi\left(E_{i}\right)^{2} .
\end{aligned}
$$

Now

$$
\begin{aligned}
\sum E_{i} E_{j} \chi\left(\left[E_{i}, E_{j}\right]\right) & =-\sum\left[E_{s}, E_{j}\right] E_{j} \chi\left(E_{s}\right) \\
& =4 \sum \chi\left(E_{s}\right)^{2}=-4 \chi(\Omega) .
\end{aligned}
$$

Thus $M^{2}=3 \chi(\Omega)=9\|\rho\|^{2}$, since $\chi$ is the sum of irreducible representations each taking the same value, $3\|\rho\|^{2}$, on $\Omega$.

The space of sections $\Gamma(\mathbf{S})$ has been identified with $C^{\infty}(G) \otimes S$. The PeterWeyl theorem decomposes $C^{\infty}(G)$, and hence $C^{\infty}(G) \otimes S$ with $\mathscr{G}$ acting via 
$\nu \otimes 1$, into the completion of a sum of representations of $G: C^{\infty}(G)=\widehat{\oplus} V_{\lambda}$ where $V_{\lambda}$ is the isotypic component of type $\lambda$. The Lie algebra $\mathscr{G}$ has a nontrivial action on $S$ via $\chi$ so that each term in the sum decomposes further: $V_{\lambda} \otimes S=\bigoplus S_{\theta}$, where $S_{\theta}$ is the isotypic component of type $\theta$. Now on $S_{\theta}$ the operator $R$ is a constant as we may see by calculating

$$
\begin{aligned}
R & =\sum \nu\left(E_{i}\right) \otimes \chi\left(E_{i}\right) \\
& =\frac{1}{2} \sum\left(\nu\left(E_{i}\right) \otimes 1+1 \otimes \chi\left(E_{i}\right)\right)^{2}-\nu\left(E_{i}\right)^{2} \otimes 1-1 \otimes \chi\left(E_{i}\right)^{2} .
\end{aligned}
$$

Hence on $S_{\theta}$

$$
\begin{aligned}
R \mid S_{\theta} & =\frac{1}{2}(-\theta(\Omega)+\lambda(\Omega)+\chi(\Omega)) \\
& =\frac{1}{2}\left(-\|\theta+\rho\|^{2}+\|\lambda+\rho\|^{2}+3\|\rho\|^{2}\right) .
\end{aligned}
$$

Since $\chi(X) M=M \chi(X)$ and $R(1 \otimes M)=(1 \otimes M) R$, the spaces $S_{\theta}$ are invariant under $M$. These spaces are also invariant under $Q$.

Proposition 2.5. When $\operatorname{rank} G>1$, (i) $\operatorname{tr} M \mid S_{\theta}=0$ and (ii) $\operatorname{tr} Q \mid S_{\theta}=0$.

Proof. (i) First, we show that $\operatorname{tr} M \mid S=0$. Let $U_{p}$ be the subspace of $\operatorname{Cliff}(G)$ spanned as a vector space by $E_{i_{1}} \cdots E_{i_{p}}, i_{1}<i_{2}<\cdots<i_{p}$. Then for $X \in U_{p}$, we have

$$
\operatorname{tr} X=0 \text { for } p \neq 0 \text {. }
$$

Now, if $\operatorname{rank} G>1$, then $\operatorname{dim} G=r>3$ and so $r-3>0$. Since $\left[E_{i}, E_{j}\right]$ is orthogonal to both $E_{i}$ and $E_{j}$, we see

$$
M=\frac{1}{4} \sum \omega E_{i}\left[E_{i}, E_{j}\right] E_{j} \in U_{r-3},
$$

and so $\operatorname{tr} M=0$.

If $\operatorname{dim} G$ is even, we replace the group $G$ by $G \times S^{1}$ and the Lie algebra $\mathscr{G}$ by $\mathscr{G} \oplus \mathbf{R}$. The space of spinors $S$ for $G$ is the same as the space of spinors for $G \times S^{1}$. Let $E$ be a unit vector in the $\mathbf{R}$ component of $\mathscr{G} \oplus \mathbf{R}$. Then the isotypic component $S_{\theta}$ for $\mathscr{G}$ decomposes under the action of $E$ into $S_{\theta}^{+} \oplus S_{\theta}^{-}$, two isotypic components for $\mathscr{G} \oplus \mathbf{R}$. Thus to show $\operatorname{tr} M \mid S_{\theta}=0$, it is sufficient if we assume $\operatorname{dim} G$ is odd.

Recall that $\chi=\Delta \circ a d$ so if we look at the weights of $\chi$ we find $\rho$ is a highest weight occurring with multiplicity $2^{n}$ where $n=\frac{1}{2}(l-1), l=\operatorname{rank} \mathscr{G}$. This follows since the weights of $\Delta$ are given by $\left\{\frac{1}{2}\left( \pm \alpha_{1} \pm \alpha_{2} \pm \cdots \pm \alpha_{r}\right)\right\}$ with $\left\{\alpha_{i}\right\}$ the simple roots of $S O(\mathscr{G})$. Thus $2^{n} V_{\rho}$ is a subspace of $S$, and since they have the same dimension this gives $S=2^{n} V_{\rho}$. Decompose $S$ into the eigenspaces of $M: S=S^{+} \oplus S^{-}$. Since $\operatorname{tr} M=0$ and $M^{2}$ is a constant $\operatorname{dim} S^{+}=\operatorname{dim} S^{-}$. As $M$ is a $G$-map, $S^{+} \cong S^{-}=2^{n-1} V_{\rho}$ so $V_{\lambda} \otimes S \cong\left(V_{\lambda} \otimes S^{+}\right) \oplus\left(V_{\lambda} \otimes S^{-}\right)$and so $V_{\lambda} \otimes S=\bigoplus S_{\theta}$ with $S_{\theta}=S_{\theta}^{+} \oplus S_{\theta}^{-}$and $\operatorname{dim} S_{\theta}^{+}=\operatorname{dim} S_{\theta}^{-}$. Thus $\operatorname{tr} M \mid S_{\theta}=0$.

(ii) With respect to the splitting $S_{\theta}=S_{\theta}^{+} \oplus S_{\theta}^{-}, M$ has matrix $\left(\begin{array}{cc}\alpha & 0 \\ 0 & -\alpha\end{array}\right)$, $\alpha=3\|\rho\|$. Let the block matrix of $Q$ with respect to this decomposition be $\left(\begin{array}{ll}A & B \\ C & D\end{array}\right)$. Then since $\{Q, 1 \otimes M\}=-6 R$

$$
\left(\begin{array}{cc}
2 \alpha A & 0 \\
0 & -2 \alpha D
\end{array}\right)=-6 \beta I
$$


where $\beta=\frac{1}{2}\left(-\|\theta+\rho\|^{2}+\|\lambda+\rho\|^{2}+3\|\rho\|^{2}\right)$ is the value of $R \mid S_{\theta}$. Since $\alpha \neq 0, A=-D$, and $\operatorname{tr} Q \mid S_{\theta}=0$.

Theorem 2.6. If rank $G>1$ and $\Gamma$ is any discrete subgroup of $G$ then $\eta_{\Gamma \backslash G}(s)=$ 0 .

Proof. Since $P=Q+\frac{1}{2}(1 \otimes M)$

$$
P^{2}=\nu(\Omega) \otimes 1-R+\frac{1}{4} M^{2} .
$$

Thus on $S_{\theta} P^{2}$ has the constant value $\alpha^{2}$ :

$$
\begin{aligned}
\alpha^{2} & =\lambda(\Omega)+\frac{1}{2} \theta(\Omega)-\frac{1}{2} \lambda(\Omega)-\frac{1}{2} \chi(\Omega)+\left(\frac{9}{4}\right)\|\rho\|^{2} \\
& =\frac{1}{2}\left(\|\theta+\rho\|^{2}+\|\lambda+\rho\|^{2}\right)-\frac{1}{4}\|\rho\|^{2} .
\end{aligned}
$$

By Proposition $2.5 \operatorname{tr} P \mid S_{\theta}=0$ and so both $\alpha$ and $-\alpha$ occur as eigenvalues of $P$ and have the same multiplicity. Over $\Gamma \backslash G$ the space of sections of $\mathbf{S}$ decomposes: $\Gamma(\mathbf{S})=\widehat{\bigoplus} V_{\lambda} \otimes S$ and $V_{\lambda} \otimes S=\bigoplus S_{\theta}$ into $G$ isotypic components with respect to the actions $\nu \otimes 1$ and $\nu \otimes \chi \mid V_{\lambda} \otimes S$. The presence of $\Gamma$ just changes the multiplicity of $\lambda$ in the isotypic component $V_{\lambda}$. Spectral symmetry on each $S_{\theta}$ yields the result of the theorem.

In fact, from this proof, we can obtain the spectrum of $P$.

Proposition 2.7. Let $\alpha$ be an eigenvalue of $P$ then

$$
\alpha^{2}=\frac{1}{2}\left(\|\theta+\rho\|^{2}+\|\lambda+\rho\|^{2}\right)-\frac{1}{4}\|\rho\|^{2} .
$$

Both eigenvalues, $\alpha$ and $-\alpha$, occur with equal multiplicity $\sum \frac{1}{2} n_{\Gamma}(\lambda, \theta) \operatorname{dim} \pi_{\theta}$, with the sum over all $\lambda$ and $\theta$ such that $\|\lambda+\rho\|^{2}+\|\theta+\rho\|^{2}=2 \alpha^{2}+\frac{1}{2}\|\rho\|^{2}$ and $n_{\Gamma}(\lambda, \theta)$ is the multiplicity of $S_{\theta}$ in $V_{\lambda} \otimes S$.

Equation (2.16) is equivalent to the Weitzenbock formula

$$
P^{2}=\nabla^{*} \nabla+\frac{1}{4} \kappa,
$$

where $\kappa$ is the scalar curvature, see [4]. By the "strange formula" of Freudenthal and deVries $\kappa=6\|\rho\|^{2}$. Comparing (2.16) and (2.18) shows they are the same if and only if

$$
\nabla^{*} \nabla=\nu(\Omega) \otimes 1-R+\frac{3}{4}\|\rho\|^{2} .
$$

This can be checked directly.

Proposition 2.8. On a compact Lie group $\nabla^{*} \nabla=\nu(\Omega) \otimes 1-R+3 / 4\|\rho\|^{2}$. Proof. Since $\nabla_{E_{i}} E_{i}=0$ in this particular case $\nabla^{*} \nabla=-\sum \nabla_{E_{i}} \nabla_{E_{i}}$. Now

$$
\nabla_{E_{i}}(f \psi)=\nu\left(E_{i}\right) f \psi+\frac{1}{2} f \chi\left(E_{i}\right) \psi
$$

and so

$$
\nabla_{E_{i}} \nabla_{E_{i}}(f \psi)=\nu\left(E_{i}\right)^{2} f \psi+\nu\left(E_{i}\right) f \chi\left(E_{i}\right) \psi+\frac{1}{4} f \chi\left(E_{i}\right)^{2} \psi
$$

Hence

$$
\nabla^{*} \nabla(f \psi)=\nu(\Omega) f \psi-\sum \nu\left(E_{i}\right) f \chi\left(E_{i}\right) \psi+\frac{1}{4} f \chi(\Omega) \psi,
$$

which is the result in the proposition since $\chi(\Omega)=3\|\rho\|^{2}$. 


\section{The DiraC OPERATOR WITH A DISCRETE ISOTROPY GROUP ACTION}

Let $G$ be a compact Lie group and let $N$ be a compact spin manifold of odd dimension. Let $G$ act on $N$ by isometries so that all the isotropy groups are discrete and such that the action lifts to an action on the spin bundle. Let $\mathscr{G}$ be the Lie algebra of $G$ and pick a basis $E_{1}, \ldots, E_{r}$ for $\mathscr{G}$ which is orthonormal with respect to the Killing form. By the formula

$$
E f(p)=\lim _{t \rightarrow 0} \frac{1}{t}(f(\exp t E p)-f(p))
$$

we can define global vector fields, also denoted by $E_{1}, \ldots, E_{r}$, on $N$. Let $V$ be the subbundle of $T(N)$ spanned by $E_{1}, \ldots, E_{r}$, so $V$ is the trivial bundle $N \times \mathscr{G}$, then this is a foliation of $N$ induced by the action of $G$. Using the metric $\rho$ of $N$, we get an orthogonal decomposition into horizontal and vertical components:

$$
T(N)=H \oplus V .
$$

Since $G$ acts by isometries, $H$ is invariant under $G$. We also require that $\rho \mid V$ gives the Killing form metric so that $E_{1}, \ldots, E_{r}$ form an orthonormal basis of vector fields for $V$. We shall use Latin subscripts to run from 1 to $r$.

For $p \in N$, there is the isotropy group $G_{p}$ and hence a $G_{p}$-slice $D$ in $N$, see [8]. The slice $D$ can be taken to be an open disc with center $p$ in a $G_{p^{-}}$ invariant subspace. Let $E_{r+1}, \ldots, E_{n}$ be an orthonormal basis of vector fields on $D$, then we can choose $D$ so $E_{r+1}, \ldots, E_{n}$ form an orthonormal basis for $H \mid D$. Using the action of $G$, we obtain vector fields $E_{r+1}, \ldots, E_{n}$ on the orbit $G D=\mathscr{U}$, an open set in $N$. Thus $\left\{E_{1}, \ldots, E_{n}\right\}$ is an orthonormal basis for $T(\mathscr{U})$. We shall use Greek subscripts to run from $r+1$ to $n$. is

The horizontal vector fields $E_{\alpha}$ are invariant by construction, see [7]. That

$$
\left[E_{i}, E_{\alpha}\right]=0 .
$$

On $N$ we use the split connection $\nabla$. This is defined on the basis $\left\{E_{1}, \ldots, E_{n}\right\}$ in the following way. First, on vertical and horizontal vectors:

$$
\begin{aligned}
\nabla_{E_{i}} E_{j} & =\frac{1}{2}\left[E_{i} E_{j}\right], \\
\nabla_{E_{\alpha}} E_{\beta} & =\frac{1}{2} \sum_{\gamma}\left(\left\langle E_{j},\left[E_{\alpha}, E_{\beta}\right]\right\rangle-\left\langle E_{\alpha},\left[E_{\beta}, E_{\gamma}\right]\right\rangle-\left\langle E_{\beta},\left[E_{\alpha}, E_{\gamma}\right]\right\rangle\right) E_{j},
\end{aligned}
$$

This $\nabla$ is the Levi-Civita connection of $G$ on vertical vectors and, in the case when $G$ acts freely, $\nabla$ on horizontal vectors is the lift of the Levi-Civita connection of $N / G$. This connection is called the split connection because it is zero on mixed expressions:

$$
\nabla_{E_{i}} E_{\beta}=\nabla_{E_{i}} E_{j}=0 .
$$

A consequence of this, which will be needed later, is the following proposition and its corollary.

Proposition 3.1. Let $X \in G$. Then, $X\left\langle E_{\alpha},\left[E_{\beta}, E_{j}\right]\right\rangle=0$.

Proof. By the Jacobi identity and (3.3), we have

$$
\left[X,\left[E_{\beta}, E_{\gamma}\right]\right]=-\left[E_{\gamma},\left[X, E_{\beta}\right]\right]-\left[E_{\beta},\left[E_{\gamma}, X\right]\right]=0 .
$$


Thus, using (3.3), we see

$$
X\left\langle E_{\alpha},\left[E_{\beta}, E_{\gamma}\right]\right\rangle=\left\langle\left[X, E_{\alpha}\right],\left[E_{\beta}, E_{\gamma}\right]\right\rangle+\left\langle E_{\alpha},\left[X,\left[E_{\beta}, E_{\gamma}\right]\right]\right\rangle=0 .
$$

Corollary 3.2. If $X \in G$, then $\nabla_{X} \nabla_{E_{\alpha}} E_{\beta}=0$.

Proof. From (3.4), and using (3.5), we see

$$
\begin{aligned}
\nabla_{X} \nabla_{E_{\alpha}} E_{\beta} & =\frac{1}{2} \nabla_{X} \sum_{\gamma}\left(\left\langle E_{\gamma},\left[E_{\alpha}, E_{\beta}\right]\right\rangle-\left\langle E_{\alpha},\left[E_{\beta}, E_{\gamma}\right]\right\rangle-\left\langle E_{\beta},\left[E_{\alpha}, E_{\gamma}\right]\right\rangle\right) E_{\gamma} \\
& =\frac{1}{2} \sum_{\gamma} X\left(\left\langle E_{\gamma},\left[E_{\alpha}, E_{\beta}\right]\right\rangle-\left\langle E_{\alpha},\left[E_{\beta}, E_{\gamma}\right]\right\rangle-\left\langle E_{\beta},\left[E_{\alpha}, E_{\gamma}\right]\right\rangle\right) E_{\gamma} .
\end{aligned}
$$

Using Proposition (3.1), we see that each of the three coefficients of $E_{\gamma}$ is zero, which proves the corollary.

The decomposition (3.2) of the tangent space gives rise to a decomposition of the Clifford algebra:

$$
\operatorname{Cliff}(T N) \cong \operatorname{Cliff}(H) \otimes \operatorname{Cliff}(V)
$$

and hence to a decomposition of the spin bundle

$$
S \cong S_{H} \otimes S_{V}
$$

Notice that since $V$ is trivial, the bundles $S_{V}$ and $\operatorname{Cliff}(V)$ are also trivial. Let $\nabla$ be a connection on $T(N)$, which preserves the metric. Then the spinor connection, also denoted by $\nabla$, on a basic spinor $\psi$ associated to elements of the basis $E_{1}, \ldots, E_{n}$ is

$$
\nabla_{X} \psi=-\frac{1}{4} \sum\left(\nabla_{X} E_{i}\right) E_{i} \psi-\frac{1}{4} \sum\left(\nabla_{X} E_{\alpha}\right) E_{\alpha} \psi
$$

where the multiplication is Clifford multiplication. Define the volume form

$$
\omega=i^{q} E_{1}, \ldots, E_{n}, q=(n+1)(n+2) / 2 ;
$$

then the Dirac operator is

$$
P=P_{V}+P_{H}
$$

where $P_{V}=\sum \omega E_{i} \nabla_{E_{i}}, P_{H}=\sum \omega E_{\alpha} \nabla_{E_{\alpha}}$, and the decomposition is independent of the bases in $H_{p}$ and $V_{p}$. The computation of the Dirac operator on any spinor can be reduced (locally) to that on spinors by the following well-known formula:

$$
P(f \psi)=\sum \nu\left(E_{i}\right) f \omega E_{i} \psi+\sum \nu\left(E_{\alpha}\right) f \omega E_{\alpha} \psi+f P \psi .
$$

For a (local) basic spinor and the split connection satisfying (3.5), we have

Lemma 3.3. $P(\psi)=-\frac{1}{4} \sum \omega E_{i}\left(\nabla_{E_{i}} E_{j}\right) \psi-\frac{1}{4} \sum \omega E_{\alpha}\left(\nabla_{E_{\alpha}} E_{\beta}\right) E_{\beta} \psi$.

We are going to prove global results by making local calculations. Fix a local basis as given above and take the corresponding basis of spinors. Define $\tilde{\chi}(X): \Gamma(S \mid U) \rightarrow \Gamma(S \mid U)$ by setting

$$
\tilde{\chi}(X) s=2 \nabla_{X} s
$$


on a basic spinor $s$ and then extending linearly over $C^{\infty}(U)$. Observe that if $X \in \mathscr{G}$, then $\tilde{\chi}(X)=1 \otimes \chi(X)$ with respect to the decomposition

$$
\Gamma(S \mid U) \cong \Gamma\left(S_{H} \mid U\right) \otimes S_{G}
$$

where $\chi$ is as in $\S 2$, that is, $\chi=\Delta \circ \widetilde{a d}$. It is also fundamental to note that there is no reason why $\tilde{\chi}\left(E_{\alpha}\right)$ should be a constant operator over $U$. As elements of $\operatorname{Cliff}(T N \mid U)$ we have the following formulae for $\tilde{\chi}(X)$ :

$$
\tilde{\chi}\left(E_{i}\right)=-\frac{1}{2} \sum\left(\nabla_{E_{i}} E_{j}\right) E_{j}, \quad \tilde{\chi}\left(E_{\alpha}\right)=-\frac{1}{2} \sum\left(\nabla_{E_{\alpha}} E_{\beta}\right) E_{\beta} .
$$

The relation $L_{X}=\nabla_{X}-\sigma(\nabla . X)$, where $\sigma: T \otimes T^{*} \rightarrow$ End $S$ and $L_{X}$ is the Lie derivative, shows that when $X \in \mathscr{G}, \tilde{\chi}(X)=L_{X}$ on basic spinors. The operator $L_{X}=\nu(X)$ acts, too, on $\Gamma\left(S_{H} \mid U\right)$ and hence we have the formula

$$
L_{X}(s \otimes \psi)=\left(L_{X} s\right) \otimes \psi+s \otimes \chi(X) \psi
$$

with respect to the decomposition (3.16). The advantage of the splitting is that operators like $\theta_{X}(s \otimes \psi)=L_{X} s \otimes \chi(X) \psi$ make global sense, and such operators will be important in our calculations. We define, locally at first, the following operators:

$$
\begin{aligned}
B & =\sum \omega E_{\alpha} \tilde{\chi}\left(E_{\alpha}\right)=-\frac{1}{2} \sum \omega E_{\alpha}\left(\nabla_{E_{\alpha}} E_{\beta}\right) E_{\beta}, \\
M & =\sum \omega E_{i} \tilde{\chi}\left(E_{i}\right)=-\frac{1}{2} \sum \omega E_{i}\left(\nabla_{E_{i}} E_{j}\right) E_{j}, \\
R & =\sum \omega_{H} L_{E_{i}} \otimes \omega_{V} \tilde{\chi}\left(E_{i}\right), \\
Q_{V} & =\sum \omega_{H} L_{E_{i}} \otimes \omega_{V} E_{i} ;
\end{aligned}
$$

where we are writing $\omega=\omega_{H} \otimes \omega_{V}$ with $\omega_{V}=i^{s} E_{1} \cdots E_{r}$ and $\omega_{H}=$ $i^{t} E_{r+1} \cdots E_{n}$ with $s=\frac{1}{2}(r+1)(r+2)$ and $t=\frac{1}{2}\{(n+1)(n+2)-(r+1)(r+2)\}$. The operators $M, R$, and $Q_{V}$ are globally defined, because every term which enters has a global meaning. The operator $B$ is not globally defined but is convenient for local calculations because it appears as the degree zero term of the operator $P_{H}$. With respect to the local decomposition $\Gamma(S \mid U) \cong C^{\infty}(U) \otimes S_{P}$ we can define $Q_{H}=\sum \nu\left(E_{\alpha}\right) \otimes \omega E_{\alpha}$. Then from Lemma 3.3 we see the following result.

Proposition 3.4. $P_{V}=Q_{V}+\frac{1}{2} M$ and $P_{H}=Q_{H}+\frac{1}{2} B$ on $L^{2}(S \mid U)$.

Let $\Delta: \operatorname{spin}(\mathscr{G}) \rightarrow$ End $S_{V}$. Then by the decomposition (3.17) $1 \otimes \Delta$ acts on $L^{2}(S)$. The following technical result is essential to our argument.

Proposition 3.5. The operator $P^{2}+R$ commutes with the $1 \otimes \Delta$ action of $\operatorname{spin}(\mathscr{G})$.

Proof. Let $X, Y \in \mathscr{G}$. Then $X$ and $Y$ act on $S_{V}$ by Clifford multiplication. Hence $X$ and $Y$ act on $L^{2}(S)$ by decomposition (3.17) with no action on $L^{2}\left(S_{H}\right)$. We shall show

$$
\left[P^{2}, X Y\right]=-[R, X Y]
$$

so $P^{2}+R$ commutes with the even part of $\operatorname{Cliff}(\mathscr{G})$ and hence with $\operatorname{spin}(\mathscr{G})$ acting via $1 \otimes \Delta$. We shall use $[A, B]=A B-B A$ for the commutator and 
$\{A, B\}=A B+B A$ for the anticommutator. A simple calculation using crucially (3.5) shows

$$
\begin{aligned}
& \left\{Q_{V}, \omega X\right\}=-2 \nu(X) \otimes 1 \in \Gamma\left(S_{H}\right) \otimes S_{G}, \\
& \left\{Q_{H}, \omega X\right\}=0, \quad\{B, \omega X\}=0, \quad\{M, \omega X\}=-6 \chi(X) .
\end{aligned}
$$

Thus, by Proposition 3.4, we have

$$
\{P, \omega X\}=-2 \nu(X) \otimes 1-3(1 \otimes \chi(X)) .
$$

Similarly a simple calculation now gives

$$
\begin{aligned}
& {\left[Q_{V}, \nu(X) \otimes 1\right]=\sum \omega_{H} \nu\left(E_{i}\right) \otimes \omega_{V}\left[X, E_{i}\right],} \\
& {\left[Q_{H}, \nu(X) \otimes 1\right]=0, \quad[B, \nu(X) \otimes 1]=0, \quad[M, \nu(X) \otimes 1]=0,}
\end{aligned}
$$

and

$$
\begin{aligned}
& {\left[Q_{V}, 1 \otimes \chi(X)\right]=\sum \omega_{H} \nu\left(E_{i}\right) \otimes \omega_{V}\left[E_{i}, X\right],} \\
& {\left[Q_{H}, 1 \otimes \chi(X)\right]=0, \quad[B, 1 \otimes \chi(X)]=0, \quad\left[M_{G}, \chi(X)\right]=0 .}
\end{aligned}
$$

To illustrate these calculations, we give the case of $[B, \nu(X) \otimes 1]$. This case was chosen because $\nabla_{E_{\alpha}} E_{\beta}$ is not a constant vector field as it is in the group case. We calculate

$$
\begin{aligned}
{[B, \nu(X) \otimes 1] } & =-\frac{1}{2}\left[\sum \omega E_{\alpha}\left(\nabla_{E_{\alpha}} E_{\beta}\right) E_{\beta}, \nu(X) \otimes 1\right] \\
& =\frac{1}{2} \sum\left\langle\nabla_{X} \nabla_{E_{\alpha}} E_{\beta}, E_{\gamma}\right\rangle \omega E_{\alpha} E_{\beta} E_{\gamma} \\
& =0
\end{aligned}
$$

by Corollary 3.2. Now since $\left[P^{2}, \omega X\right]=[P,\{P, \omega X\}]$ and by Proposition 3.4 and equation (3.22) we have

$$
\left[P^{2}, \omega X\right]=\sum \nu\left(E_{i}\right) \otimes \omega\left[X, E_{i}\right] .
$$

Using the fact

$$
-\left[\chi\left(E_{i}\right), X Y\right]=\left[X, E_{i}\right] Y+X\left[Y, E_{i}\right]
$$

we calculate

$$
\begin{aligned}
{\left[P^{2}, X Y\right] } & =\left[P^{2}, \omega X\right] \omega Y+\omega X\left[P^{2}, \omega Y\right] \\
& =\sum \nu\left(E_{i}\right) \otimes \omega\left(\left[X, E_{i}\right] Y+X\left[Y, E_{i}\right]\right) \\
& =-\sum \nu\left(E_{i}\right) \otimes \omega\left[\chi\left(E_{i}\right), X Y\right] \\
& =-[R, X Y] .
\end{aligned}
$$

This completes the proof of Proposition 3.5.

\section{THE CASE OF AN ABELIAN GROUP ACTION}

Let $T^{l}$ act on $N$ with discrete isotropy subgroups. Let $\left\{E_{1}, \ldots, E_{l}\right\}$ be an orthonormal basis for the Lie algebra $\mathscr{T}$ of $T^{l}$; otherwise, we keep the notation of $\S 3$. The calculations of $\S 3$ still hold, and we see that

$$
R=M=0 .
$$

Let $E=E_{1} \cdots E_{l-1}$ and $F=E_{l}$. Then, we have the following calculation. 
Lemma 4.1. (i) $\left\{\omega_{H} E, B\right\}=0$, (ii) $\left\{\omega_{H} E, Q_{H}\right\}=0$, (iii) $\left\{\omega_{H} E, Q_{V}\right\}=$ $2 L_{F}$.

Proof. These are all obtained by a direct calculation using the definitions of $B, Q_{H}$, and $Q_{V}$ and the following formulae:

$$
\omega E_{\alpha}=E_{\alpha} \omega, \quad \omega E_{i}=E_{i} \omega, \quad \omega^{2}=1 .
$$

As an illustration we shall give the proof of part (iii). First, notice that

$$
E=E_{1} \cdots E_{l-1}=-\omega_{V} F .
$$

Thus, we calculate, as in equation (3.21):

$$
\begin{aligned}
\left\{\omega_{H} E, Q_{V}\right\} & =-\left\{\omega F, Q_{V}\right\} \\
& =-\sum L_{E_{i}} \otimes\left(\omega F \omega E_{i}+\omega E_{i} \omega F\right) \\
& =-\sum L_{E_{i}} \otimes\left(F E_{i}+E_{i} F\right)=2 L_{F} .
\end{aligned}
$$

Proposition 4.2. $\left\{\omega_{H} E, P\right\}=2 L_{F} \otimes 1$.

Proof. By Proposition 3.4 $P=Q_{H}+Q_{V}+\frac{1}{2} B$ and the result follows from Lemma 4.1.

Corollary 4.3. $\left[P^{2}, \omega_{H} E\right]=0$.

Proof. We calculate

$$
\left[P^{2}, \omega_{H} E\right]=\left[P,\left\{P, \omega_{H} E\right\}\right]=2\left[P, L_{F} \otimes 1\right]=0
$$

since $P$ is an invariant operator.

Now we decompose $L^{2}(S)=\sum V_{\lambda}$ into eigenspaces of $P^{2}$ and further decompose $V_{\lambda}$ under the action of $T^{l}$ :

$$
V_{\lambda}=\sum S_{\theta, \lambda}
$$

Since $L_{F}$ acts as a constant on any irreducible representation space of $T^{l}$, we find the following:

Lemma 4.4. (i) $\left\{\omega_{H} E, P\right\}$ is constant on $S_{\theta, \lambda}$, (ii) $\operatorname{tr} \omega_{H} E \mid S_{\theta, \lambda}=0$.

Proof. Part (i) is immediate. We decompose $S_{T}=S_{T}^{+} \oplus S_{T}^{-}$into eigenspaces of $i \omega_{V}$. Then $V_{\lambda}=V_{\lambda}^{H} \otimes S_{T} \cong V_{\lambda}^{H} \otimes\left(S_{T}^{+} \oplus S_{T}^{-}\right)$and $\omega_{H} E$ interchanges $V_{\lambda}^{+}=V_{\lambda}^{H} \otimes S_{T}^{+}$and $V_{\lambda}^{-}=V_{\lambda}^{H} \otimes S_{T}^{-}$.

Theorem 4.5. On $S_{\theta, \lambda}, P$ has two eigenspaces $\mu$ and $-\mu$ which have the same multiplicity.

Proof. Decompose $S_{\theta, \lambda}=S_{\theta, \lambda}^{+} \oplus S_{\theta, \lambda}^{-}$into eigenspaces of $\omega_{H} E$. Then, $\operatorname{dim} S_{\theta, \lambda}^{+}=\operatorname{dim} S_{\theta, \lambda}^{-}$and relative to this decomposition $\omega_{H} E$ and $P$ have the following block form:

$$
\omega_{H} E=\left(\begin{array}{cc}
1 & 0 \\
0 & -1
\end{array}\right), \quad P=\left(\begin{array}{ll}
a & b \\
c & d
\end{array}\right) .
$$

Thus $\left\{\omega_{H} E, P\right\}=\left(\begin{array}{cc}2 a & 0 \\ 0 & -2 d\end{array}\right)$ and, since this is constant, $a=-d$ and $\operatorname{tr} P \mid S_{\theta, \lambda}$ $=0$. 
Now $P^{2}$ is constant on $S_{\theta, \lambda}$, and therefore, $P$ has two eigenvalues $\mu$ and $-\mu$ on $S_{\theta, \lambda}$ where $P^{2}=\mu^{2} I$ on $S_{\theta, \lambda}$. The trace of $P$ is given by

$$
\operatorname{tr} P \mid S_{\theta, \lambda}=\mu(\operatorname{mult}(\mu)-\operatorname{mult}(-\mu)) .
$$

Since this is zero, $\operatorname{mult}(\mu)=\operatorname{mult}(-\mu)$.

\section{THE CASE OF A NONABELIAN GROUP ACTION}

Let $G$ be a compact nonabelian Lie group with $\operatorname{rank} G \geq 2$ acting on $N$ by isometries with discrete isotropy. We keep the same notation as in $\S 3$, and observe that $M$ and $R$ are nonzero in this case. While this prevents the proof for the abelian case from carrying over directly, we can use $M$ in place of $\omega_{H} E$ to give a proof in this case.

Now decompose $L^{2}(S)=\sum V_{\lambda}$ into eigenspaces of $P^{2}+R$. Since $P^{2}$ is an elliptic second order operator and $R$ is a first order operator $P^{2}+R$ is a second order operator. This gives that the spaces $V_{\lambda}$ are finite-dimensional and since $P^{2}+R$ commutes with the $1 \otimes \Delta$ action of $\operatorname{spin}(\mathscr{G})$ we obtain

Theorem 5.1. $V_{\lambda}=V_{\lambda}^{H} \otimes S_{G}$ for a finite-dimensional space $V_{\lambda}^{H}$.

The proofs of the next three lemmas are similar to those of $\S 2$.

Lemma 5.2. $\operatorname{tr} M_{V}=0$.

Lemma 5.3. $M^{2}=9\|\rho\|^{2}$.

Proof. Since $n$ is odd $\omega_{H} \omega_{V}=\omega_{V} \omega_{H}=\omega$ is a decomposition of the volume form. Using the decomposition (3.16) we see that $M$ acts on $L^{2}\left(S_{H}\right) \otimes S_{G}$ by

$$
M=\omega_{H} \otimes M_{G} .
$$

Now the powers of $i$ in the volume forms have been chosen so $\omega^{2}=1, \omega_{H}^{2}=$ $(-1)^{n-r}$, and $\omega_{V}^{2}=(-1)^{r-1}$. Thus, $M^{2}=\omega_{H}^{2} \otimes(-1)^{3(n-r)} M_{G}^{2}=M_{G}^{2}$, and the result follows from the group case, see $\S 2$. Of course $\rho=\frac{1}{2} \sum_{\alpha>0} \alpha$ is half the sum of the positive roots of $G$.

Lemma 5.4. Decompose $S_{G}=S_{G}^{+} \oplus S_{G}^{-}$into eigenspaces of $M_{G}$, then $\operatorname{dim} S_{G}^{+}=$ $\operatorname{dim} S_{G}^{-}$and, as representation spaces of $\mathscr{G}, S_{G}^{+} \cong S_{G}^{-} \cong 2^{k-1} V_{\rho}$.

Proof. Since $M_{G}^{2}$ is constant and $\operatorname{tr} M_{G}=0$, we have immediately that there are only two eigenspaces and these have equal dimensions. If $\operatorname{rank} G=l$ and $k=\frac{1}{2}(l-1)$ (for $l$ odd) or $k=\frac{1}{2} l$ (l even), then $S_{G}=2^{k} V_{\rho}$. Thus, as representation spaces of $\mathscr{G}, S_{G}^{+}$and $S_{G}^{-}$consist of a number of copies of $V_{\rho}$. Counting dimensions determines that there are $2^{k-1}$ copies, so $S_{G}^{+} \cong S_{G}^{-} \cong$ $2^{k-1} V_{\rho}$.

Now decompose $V_{\lambda}=\sum S_{\theta, \lambda}$ into isotypic components under the action of $\mathscr{G}$ by $\chi$. We shall show that $P$ has spectral symmetry on each $S_{\theta, \lambda}$ by showing $\operatorname{tr} P \mid S_{\theta, \lambda}=0$ and $P^{2} \mid S_{\theta, \lambda}$ is constant. First there is a lemma.

Lemma 5.5. $\operatorname{tr} M \mid S_{\theta, \lambda}=0$.

Proof. We have $V_{\lambda}=V_{\lambda}^{H} \otimes S_{G}$ and $S_{G}=S_{G}^{+} \oplus S_{G}^{-}$so that

$$
V_{\lambda}=\left(V_{\lambda}^{H} \otimes S_{G}^{+}\right) \oplus\left(V_{\lambda}^{H} \otimes S_{G}^{-}\right) .
$$


Thus since $S_{G}^{+} \cong S_{G}^{-}$as representation spaces

$$
S_{\theta, \lambda}=S_{\theta, \lambda}^{+} \oplus S_{\theta, \lambda}^{-}
$$

and $S_{\theta, \lambda}^{+} \cong S_{\theta, \lambda}^{-}$as representation spaces and the lemma is proved.

Proposition 5.6. $\left\{M, P_{V}\right\}=M P_{V}+P_{V} M=-6 R+9\|\rho\|^{2}$ is constant on $S_{\theta, \lambda}$ and $\left\{M, P_{H}\right\}=0$.

Proof. By Proposition 3.4, $P=Q_{V}+Q_{H}+\frac{1}{2} B+\frac{1}{2} M$. Thus the proof is complete when we show the following four operators are constant: (1) $\left\{M, Q_{V}\right\}$, (2) $\left\{M, Q_{H}\right\},(3)\{M, B\}$, and (4) $\{M, M\}$. A direct calculation gives

$$
\begin{gathered}
\left\{M, Q_{V}\right\}=-6 R, \quad\left\{M, Q_{H}\right\}=0, \\
\{M, B\}=0, \quad\{M, M\}=2 M^{2}=18\|\rho\|^{2} .
\end{gathered}
$$

To complete the proof we have the following lemma showing $R \mid S_{\theta, \lambda}$ is constant.

Lemma 5.7. $R \mid S_{\theta, \lambda}$ is constant.

Proof. First a calculation:

$$
\begin{aligned}
R & =\sum \omega_{H} L_{E_{i}} \otimes \omega_{V} \chi\left(E_{i}\right) \\
& =-\frac{1}{2}((\nu \otimes 1+1 \otimes \chi)(\Omega)-\nu(\Omega) \otimes 1-1 \otimes \chi(\Omega)),
\end{aligned}
$$

where $\Omega$ is the Casimir element of $\mathscr{G}$. Thus $R$ is constant on irreducible representations of $\mathscr{G}$ and the constant depends only on the representation. Thus $R$ is constant on $S_{\theta, \lambda}$.

Proposition 5.8. If $A: S_{\theta, \lambda} \rightarrow S_{\theta, \lambda}$ is any operator such that $\{M, A\}$ is constant, then $\operatorname{tr} A=0$.

Proof. Decompose $S_{\theta, \lambda}=S_{\theta, \lambda}^{+} \oplus S_{\theta, \lambda}^{-}$into (5.3). Then $\operatorname{dim} S_{\theta, \lambda}^{+}=\operatorname{dim} S_{\theta, \lambda}^{-}$ and relative to this decomposition, $M$ and $A$ have the following block form:

$$
M=\left(\begin{array}{cc}
\alpha & 0 \\
0 & -\alpha
\end{array}\right), \quad A=\left(\begin{array}{ll}
a & b \\
c & d
\end{array}\right),
$$

with $\alpha=3\|\rho\| \neq 0$. Thus $\{M, A\}=\left(\begin{array}{cc}2 \alpha a & 0 \\ 0 & -2 \alpha d\end{array}\right)$ and since this is constant $a=-d$ and $\operatorname{tr} A=0$.

Corollary 5.9. $\operatorname{tr} P \mid S_{\theta, \lambda}=0$.

Theorem 5.10. On $S_{\theta, \lambda}, P$ has two eigenvalues $\mu$ and $-\mu$ and these have the same multiplicity.

Proof. Since $S_{\theta, \lambda} \subset V_{\lambda}, P^{2}+R$ is constant on $S_{\theta, \lambda}$. By Lemma 5.7, $R$ is constant on $S_{\theta, \lambda}$ and so $P^{2}$ is constant on $S_{\theta, \lambda}$. Thus $P$ has two eigenvalues $\mu$ and $-\mu$ on $S_{\theta, \lambda}$, where $P^{2}=\mu^{2} I$ on $S_{\theta, \lambda}$. The trace of $P$ is given by

$$
\operatorname{tr} P \mid S_{\theta, \lambda}=\mu(\operatorname{mult}(\mu)-\operatorname{mult}(-\mu)) \text {. }
$$

Since this is zero, $\operatorname{mult}(\mu)=\operatorname{mult}(-\mu)$.

Corollary 5.11. For each $\mu$ the eigenspaces of the Dirac operator corresponding to $\mu$ and $-\mu$ are G-isomorphic.

\section{THE CHANGE OF CONNECTION}

We start by defining the difference tensor

$$
S(X, Y)=\widetilde{\nabla}_{X} Y-\nabla_{X} Y
$$


where $\widetilde{\nabla}$ is a connection on $N$ which preserves the metric, $\nabla$ is the split connection of $\S 3$, and $X$ and $Y$ are vector fields. Using this, we can obtain the following.

Proposition 6.1. $\widetilde{P}=P+T$ where $\widetilde{P}$ is the Dirac operator defined using $\widetilde{\nabla}, P$ is the split Dirac operator and $T$ is Clifford multiplication by

$$
\begin{aligned}
& -\frac{1}{4} \sum \omega E_{i} S\left(E_{i}, E_{j}\right) E_{j}-\frac{1}{4} \sum \omega E_{i} S\left(E_{i}, E_{\beta}\right) E_{\beta} \\
& -\frac{1}{4} \sum \omega E_{\alpha} S\left(E_{\alpha}, E_{j}\right) E_{j}-\frac{1}{4} \sum \omega E_{\alpha} S\left(E_{\alpha}, E_{\beta}\right) E_{\beta} .
\end{aligned}
$$

Proof. First, observe that by using (3.14) it is sufficient to prove

$$
\widetilde{P} \psi=P \psi+T \psi
$$

when $\psi$ is a basic spinor. On basic spinors, we have

$$
\begin{aligned}
\widetilde{P} \psi= & -\frac{1}{4} \sum \omega E_{i}\left(\widetilde{\nabla}_{E_{i}} E_{j}\right) E_{j} \psi-\frac{1}{4} \sum \omega E_{i}\left(\widetilde{\nabla}_{E_{i}} E_{\beta}\right) E_{\beta} \psi \\
& -\frac{1}{4} \sum \omega E_{\alpha}\left(\widetilde{\nabla}_{E_{\alpha}} E_{j}\right) E_{j} \psi-\frac{1}{4} \sum \omega E_{\alpha}\left(\widetilde{\nabla}_{E_{\alpha}} E_{\beta}\right) E_{\beta} \psi \\
= & P \psi+T \psi . \quad \square
\end{aligned}
$$

In the case when $\tilde{\nabla}=\nabla^{L}$ is the Levi-Civita connection, these expressions simplify. Let $P^{L}$ denote the Dirac operator defined using the Levi-Civita connection.

Proposition 6.2. (i) $S^{L}\left(E_{i}, E_{j}\right)=0$,

(ii) $S^{L}\left(E_{i}, E_{\alpha}\right)=-\frac{1}{2} \sum_{\beta}\left\langle E_{i},\left[E_{\alpha}, E_{\beta}\right]\right\rangle E_{\beta}$,

(iii) $S^{L}\left(E_{\alpha}, E_{i}\right)=-\frac{1}{2} \sum_{\beta}\left\langle E_{i},\left[E_{\alpha}, E_{\beta}\right]\right\rangle E_{\beta}$,

(iv) $S^{L}\left(E_{\alpha}, E_{\beta}\right)=\frac{1}{2} \sum_{j}\left\langle E_{j},\left[E_{\alpha}, E_{\beta}\right]\right\rangle E_{j}$.

Proof. For any three orthonormal vector fields, the Levi-Civita connection satisfies

$$
\left\langle\nabla_{X}^{L} Y, Z\right\rangle=\frac{1}{2}(\langle Z,[X, Y]\rangle-\langle X,[Y, Z]\rangle-\langle Y,[X, Z]\rangle) .
$$

Using (3.4), (3.5), and (6.4), the results of Proposition 6.2 follow immediately.

The result of Proposition 6.1 is now simplified considerably.

Theorem 6.3. $P^{L}=P+\frac{1}{4} \sum_{\alpha, \beta} \omega\left[E_{\alpha}, E_{\beta}\right]^{v} E_{\alpha} E_{\beta}$, where the superscript $v$ denotes the vertical part.

Proof. This requires that we calculate $T^{L}=T$ for the Levi-Civita connection:

$$
\begin{aligned}
T^{L}= & -\frac{1}{4} \sum \omega E_{i} S\left(E_{i}, E_{j}\right) E_{j}-\frac{1}{4} \sum \omega E_{i} S\left(E_{i}, E_{\alpha}\right) E_{\alpha} \\
& -\frac{1}{4} \sum \omega E_{\alpha} S\left(E_{\alpha}, E_{i}\right) E_{i}-\frac{1}{4} \sum \omega E_{\alpha} S\left(E_{\alpha}, E_{\beta}\right) E_{\beta} \\
= & \frac{1}{4} \sum \omega\left[E_{\alpha}, E_{\beta}\right]^{v} E_{\alpha} E_{\beta} .
\end{aligned}
$$

This last step follows since

$$
\begin{aligned}
E_{i} S\left(E_{\alpha}, E_{i}\right) & =-S\left(E_{\alpha}, E_{i}\right) E_{i}, \quad E_{\alpha} S\left(E_{\alpha}, E_{i}\right)=-S\left(E_{\alpha}, E_{i}\right) E_{\alpha} \\
S\left(E_{i}, E_{\alpha}\right) & =S\left(E_{\alpha}, E_{i}\right), \quad \text { and } \quad S\left(E_{\alpha}, E_{\beta}\right)=\left[E_{\alpha}, E_{\beta}\right]^{\prime} .
\end{aligned}
$$


Let $\eta$ denote the eta function of $P$ and $\tilde{\eta}$ denote the eta function of $\widetilde{P}$. Then, if we define

$$
X=N \times[0,1], \quad P_{t}=P+t T, \quad \text { and } \quad D=P_{t}+\partial / \partial t,
$$

thus, if $P_{t}$ is an operator on $N$ with $\widetilde{P}=P_{1}$ and $D$ is an operator on $N \times I$, we can use the result of [2]:

$$
\text { index } D=\int_{N \times I} \alpha_{0}-\frac{(\tilde{\eta}(0)-\eta(0))+h}{2} .
$$

By Corollary 5.11, we have $\eta(0)=0$. Since the adjoint of $D$ is

$$
D^{*}=P_{t}-\partial / \partial t
$$

it follows that index $D=0$. This is because, if $D u=0$, set

$$
v(n, t)=u(n, 1-t),
$$

then, $D^{*} v=0$. Thus $\operatorname{ker} D \cong \operatorname{ker} D^{*}$ as $G$ spaces, that is, the $G$-index $D=0$. Let $H=\operatorname{ker} P$ and $\widetilde{H}=\operatorname{ker} \widetilde{P}$ be the spaces of harmonic spinors. Then, these are $G$-spaces, and we define the characters

$$
H(g)=\operatorname{tr}(g \mid H) \text { and } \widetilde{H}(g)=\operatorname{tr}(g \mid \widetilde{H}) .
$$

With this definition, $h$ is the difference

$$
h(g)=\tilde{H}(g)-H(g) .
$$

Now $\alpha_{0}$ is the constant term in the asymptotic expansion as $t \rightarrow 0$ of

$$
\operatorname{tr} e^{-t D^{*} D}-\operatorname{tr} e^{-t D D^{*}} \text {. }
$$

Since $G$ acts by isometries, the action of $G$ commutes with both $P_{0}$ and $P_{1}$. Hence, the action of $G$ commutes with $T$, and therefore, with $D$ and $D^{*}$. Thus $\alpha_{0}$ is constant and does not depend on $g$. If we substitute these into (6.8), we obtain the following result.

Theorem 6.4. Let $\tilde{\eta}_{G}(0, g)$ be the equivariant eta invariant of the Dirac operator $\widetilde{P}$. Then, $\tilde{\eta}_{G}(0, g)=a+\widetilde{H}(g)-H(g)$ where $a=2 \int_{N \times i} \alpha_{0}$ is constant.

Corollary 6.5. Reduced $\bmod R(G)$, that is, as an element of $R(G) \otimes \mathbf{R} / R(G)$, $\tilde{\eta}_{G}(o, g)$ is constant.

\section{APPendix. The Rank ONE CASE}

The results of this paper sometimes fail to hold in the rank one case. The main difference in the rank one case, and the reason for this failure, is that our operator $M$ is a constant multiple of 1 . Those symmetry results which are still true, hold for different reasons. As an example consider the group $S U(2)$. For convenience we renormalize the metric (by a factor 8) so that $\|\rho\|^{2}=1$. The basic results in this case can be found in [5] and so we state them without proof.

The irreducible representations of $S U(2), V_{n}$, are indexed by the positive integers, $\{n\}$, with $\operatorname{dim} V_{n}=n$. The spin module is $S \cong \mathbf{C}^{2}$ and $V_{n} \otimes S=$ $V_{n+1} \oplus V_{n-1}$ with respect to the $n \otimes \chi$ action. From [5] we see that

$$
M=3 I
$$


and

$$
P=Q+\frac{3}{2} I
$$

The matrix of $Q$ relative to the splitting of $V_{n} \otimes S$ is

$$
Q=\left(\begin{array}{cc}
n-1 & 0 \\
0 & -n-1
\end{array}\right)
$$

It is now an easy matter to read off the spectrum of both $Q$ and $P$.

Proposition A.1. The spectrum of $Q$ is eigenvalue $n-1$ with multiplicity $n+1$ and eigenvalue $-n-1$ with multiplicity $n-1$. The spectrum of $P$ is eigenvalue $n+\frac{1}{2}$ with multiplicity $n+1$ and eigenvalue $-n+\frac{1}{2}$ with multiplicity $n-1$.

Corollary A.2. When restricted to the space $V_{n} \otimes S$ the operators have the following traces:

$$
\operatorname{tr} Q\left|V_{n} \otimes S=0, \quad \operatorname{tr} M\right| V_{n} \otimes S=6 n, \quad \operatorname{tr} P \mid V_{n} \otimes S=2 n .
$$

Thus, we see that the operator $P$ does not have spectral symmetry on $V_{n} \otimes S$. However, the multiplicity of the eigenvalue $-n+\frac{1}{2}$ is the same as that of the eigenvalue $n-\frac{1}{2}=(n-1)+\frac{1}{2}$, namely $n-1$. We see, for different reasons from those in the rest of this paper, that $P$ has spectral symmetry.

The spectral symmetry of the Dirac operator on $S U(2)$ is more subtle than in the higher rank cases and can fail on $\Gamma \backslash S U(2)$ for a discrete subgroup $\Gamma$. To see this consider $\Gamma=\{I,-I\}$ so $\Gamma \backslash S U(2)=S O(3)$. While it is possible, in principle, to calculate the eta invariant for $S O(3)$ by using the complete knowledge of the spectrum of $P$ on $S U(2)$ it is very much easier to use the index theorem of [2]. This gives, for example, $\eta_{P}(0)=-1 / 8$ when we take the trivial spin structure on $S O(3)$.

A simple argument for spectral symmetry on a compact group $G$ is to consider the map $x \mapsto x^{-1}$. If its derivative lifts to a map of spin bundles, there is spectral symmetry. This is so for the trivial spin structure if and only if ad: $G \rightarrow S O(\mathscr{G})$ lifts to Spin. In the case of $S O(3)$ the adjoint map does not lift.

The case of a noncompact rank one group is discussed in [10] and the reader is referred there for details of this case.

\section{REFERENCES}

1. M. F. Atiyah and F. Hirzebruch, Spin manifolds and group actions, Essays on Topology and Related Topics, Memoires dédiés à George deRham (A. Haefliger and R. Narashiman, eds.), Springer-Verlag, 1970, pp. 18-28.

2. M. F. Atiyah, V. K. Patodi, and I. M. Singer, Spectral asymmetry and Riemannian geometry. I and II, Math. Proc. Cambridge Philos. Soc. 77 (1975), 43-69 and 78 (1976), 405-432.

3. H. D. Fegan and B. Steer, On the "strange formula" of Freudenthal and de Vries, Math. Proc. Cambridge Philos. Soc. 105 (1989), 249-252.

4. M. Gromov and H. B. Lawson, Spin and scalar curvature in the presence of a fundamental group. I, Ann. of Math. 111 (1980), 209-230.

5. N. Hitchin, Harmonic spinors, Adv. in Math. 14 (1974), 1-55.

6. K. H. Knapp, Rank and Adams filtration of a Lie group, Topology 17 (1978), 41-52.

7. H. B. Lawson and S. T. Yau, Scalar curvature non-abelian group actions and the degree of symmetry of exotic spheres, Comment Math. Helv. 49 (1974), 232-244. 
8. R. S. Palais, Slices and equivariant imbeddings, Seminar on Transformation Groups (by A. Borel), Princeton Univ. Press, Princeton, N. J., 1960.

9. R. Parthasarathy, Dirac operators and the discrete series, Ann. of Math. 96 (1972), 1-30.

10. J. A. Seade and B. Steer, A note on the eta-function for quotients of $P S L_{2}(\mathbf{R})$ by co-compact Fuchsian groups, Topology 26 (1987), 79-91.

11. S. Slebarski, Dirac operators on a compact Lie group, Bull. London Math. Soc. 17 (1985), 579-583.

Department of Mathematics, University of New Mexico, Albuquerque, New Mexico 87131

Hertford College, OXford, OX1 3BR, England 